\title{
Feminicidios de género: Evolución real del fenómeno, el suicidio del agresor y la incidencia del tratamiento mediático.
}

Manuscrito recibido el 16 de marzo de 2011 / Publicado en junio de 2011

\author{
Javier Gustavo Fernández Teruelo ${ }^{1}$ \\ Universidad de Oviedo
}

\begin{abstract}
RESUMEN
En este trabajo se analizan tres de las cuestiones que actualmente acaparan mayor preocupación social y científica en el ámbito de la violencia de género en general y de los denominados feminicidios de género (muerte violenta de la mujer ejecutada por su pareja) en particular. Una primera parte se dedica a tratar de valorar, más allá de lo que puede apreciarse con el mero examen de la evolución del número de casos, al análisis, mediante factores correctores que ayudan a entender el fenómeno, de la evolución real de los feminicidios de género en la última década. En segundo lugar, se trata de profundizar sobre uno de los fenómenos más llamativos; se trata del suicidio (consumado o tentado) del agresor tras dar muerte a su pareja, que en las últimas estadísticas se aproxima ya a un tercio de los casos. Finalmente, se analiza si la distribución de feminicidios de género sigue un patrón uniforme para comprobar si se puede estar produciendo el denominado "efecto imitación o llamada"; esto es que determinados sujetos ejecutan o aceleran la ejecución de un feminicidio de género al tener conocimiento por los medios de comunicación de que otros, en una situación similar a la suya, lo han llevado a cabo.
\end{abstract}

Palabras clave: Feminicidio, violencia de género, medios de comunicación.

\footnotetext{
${ }^{1}$ Emilio Torres Manzanera (profesor Titular de estadística en la Universidad de Oviedo) es el autor del análisis estadístico contenido en el apartado 3.
} 


\begin{abstract}
Throughout this article discussed three issues that currently account for most social and scientific concern in the field of gender violence in general and so-called femicide (violent death of the woman executed by her partner) in particular. The first part is focused on trying to assess, beyond what can be seen by simply examining the real evolution of the number of cases, the analysis by applying correcting factors that help to understand the phenomenon, of evolution of gender femicide in the last decade. Second, is to deepen on one of the most striking facts, as it is suicide (accomplished or attempted) of the aggressor after killing his partner that in the latest data now approaches one third of total cases. Finally it also analyses whether the distribution of femicide follows a uniform pattern to determine if it could be producing the so-called "imitation or call effect" That, means certain individuals carry out or accelerate the implementation of a gender femicide when getting through media, that others in a similar situation have done so.
\end{abstract}

Keywords: Femicide, violence against women, mass media.

\title{
1. Introducción
}

En la primera parte dedicada al análisis de la evolución real de las tasas de feminicidios de género, pondremos de manifiesto la imperiosa necesidad de introducir factores correctores en la valoración pues, sólo sobre el resultado de tal operación, podrán hacerse las valoraciones sobre la mayor o menor eficacia de las medidas de acción que, en todos los órdenes, se han adoptado en la última década frente a la violencia de género.

En la segunda parte del trabajo, se reflexiona sobre los factores que influyen en la decisión suicida del agresor tras cometer el feminicidio y ello fundamentalmente con dos objetivos: en primer lugar, para tratar de comprender mejor la violencia de género extrema y reflexionar sobre la nula o escasa eficacia de políticas legislativas penales que pretenden la intimidación del agresor; un agresor que, sin embargo resulta inasequible a cualquier tipo de amenaza y, entre ellas, por supuesto a las sanciones penales.

Finalmente, el tercer aspecto analizado, relativo a la existencia o inexistencia del efecto "llamada" o "estímulo", sirve para centrar el debate sobre si deben (o no) establecerse límites en el modo de tratamiento de los feminicidios de género por parte de los medios de comunicación. 


\section{Procedimiento}

En el desarrollo del trabajo se analiza y reflexiona sobre los datos conocidos y reflejados gráficamente relativos a la evolución del número de feminicidios, complementados con variables que no suelen ser tenidos en cuenta, como la nacionalidad del agresor y en particular el porcentaje de extranjeros autores del referido comportamiento. También se cruza la información relativa al número de denuncias para analizar evoluciones comparativas entre ambos factores.

En la parte relativa a la evaluación del suicidio del agresor, el esfuerzo se ha centrado en contrastar las conclusiones derivadas de la experiencia investigadora anglosajona sobre el Homicidio-Suicidio (H/S) con las informaciones y datos con los que contamos y que ayudan a conformar el perfil del que denominamos Feminicidio-Suicidio (F/S).

En la tercera parte del trabajo se recurre a un análisis fundamentalmente estadístico para tratar de demostrar la existencia o inexistencia del "efecto llamada" o "efecto estimulador", que se ve acompañada de reflexiones sobre cada uno de los puntos analizados. En concreto, se analizó la información recogida por el Centro Reina Sofía, denominación de la Fundación de la Comunidad Valenciana para el Estudio de la Violencia $^{2}$. En el mismo se presenta de forma sistematizada la información más relevante en relación con mujeres muertas por violencia de género en España y proporciona el día del suceso, el medio material empleado y lo que aconteció con el agresor. El periodo de estudio abarca desde enero del 2005 hasta abril de 2010, ambos inclusive. Se desecharon 7 registros por la escasa información disponible e incorporaron los intentos (tentativas) de feminicidio recogidos.

Se realizó un análisis descriptivo de los datos y un contraste de independencia con el test $\chi^{2}(\alpha=0.05)$ (Lebart, L., Morineau, A. and Piron, M., 1995). Para comprobar la estacionalidad mensual, se efectuó un análisis de la autocorrelación y de la autocorrelación parcial de la serie de datos mensuales, así como un test de KruskalWallis para los datos mensuales y un test $\chi^{2}$ para verificar la distribución aleatoria de los datos semanales. Se analizaron los lapsos de tiempo entre feminicidios consecutivos y se contrastó la aleatoriedad o no de la distribución de los feminicidios mediante el test de Kolmogorov-Smirnoff para una distribución uniforme.

\footnotetext{
${ }^{2} \mathrm{http} / /$ www.centroreinasofia.es/consultafemicidios.asp?id=36 [Consulta 28-01-2011].
} 
Por último, se estudió la distribución secuencial diaria mediante un análisis de la autocorrelación y la autocorrelación parcial del número de fallecidas. Dado que esta variable toma valores de 0 a 3 , se creó una variable dicotómica 0-1 indicando si en el día correspondiente hubo feminicidio. Esta variable se relacionó con las series retardadas desde uno hasta 30 días mediante un modelo logit. Se ha utilizado el paquete estadístico R 2.10

\section{La evolución real de las tasas de violencia de género (denuncias y feminicidios)}

Una correcta valoración de la evolución de todos los indicadores relacionados con el fenómeno de la violencia de género (víctimas de maltrato, muertes, etc.) precisa el análisis de periodos de tiempo prolongados; sin embargo, podemos comprobar cómo, con frecuencia, se realizan valoraciones sobre la evolución de este fenómeno, utilizando únicamente los cambios producidos en periodos muy breves, de los cuales se pretende extraer sin embargo conclusiones definitivas Por ejemplo, de acuerdo con estos parámetros, encontramos afirmaciones según las cuales en el año 2010 se habría producido un notable incremento en las tasas de violencia de género extrema (feminicidios de género); sin embargo, ello sólo es así, si se compara ese año con el anterior en el que, por cierto, se produjo una reducción notable respecto a los anteriores. Es, por contra, precisa una valoración a medio/largo plazo y sólo entonces podemos sacar conclusiones sobre la real evolución del fenómeno, pues estamos analizando guarismos singularmente bajos (desde un punto de vista estadístico).

[FIGURA 1. Feminicidios de género (1995-1997): Fuente gráfica: Informe del Defensor del Pueblo. Ministerio del Interior 1998]

\section{MUERTES DE MUJERES A MANOS DE SUS CONYUGES O COMPAÑEROS}

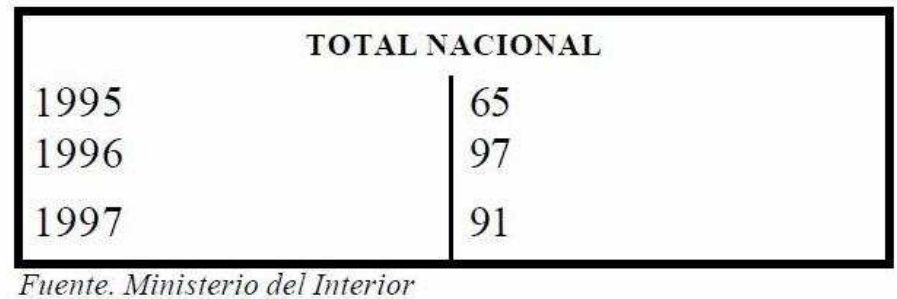


[FIGURA 2. Feminicidios de género (2000-2009): Fuente gráfica: Centro Reina Sofía para el estudio de la violencia]

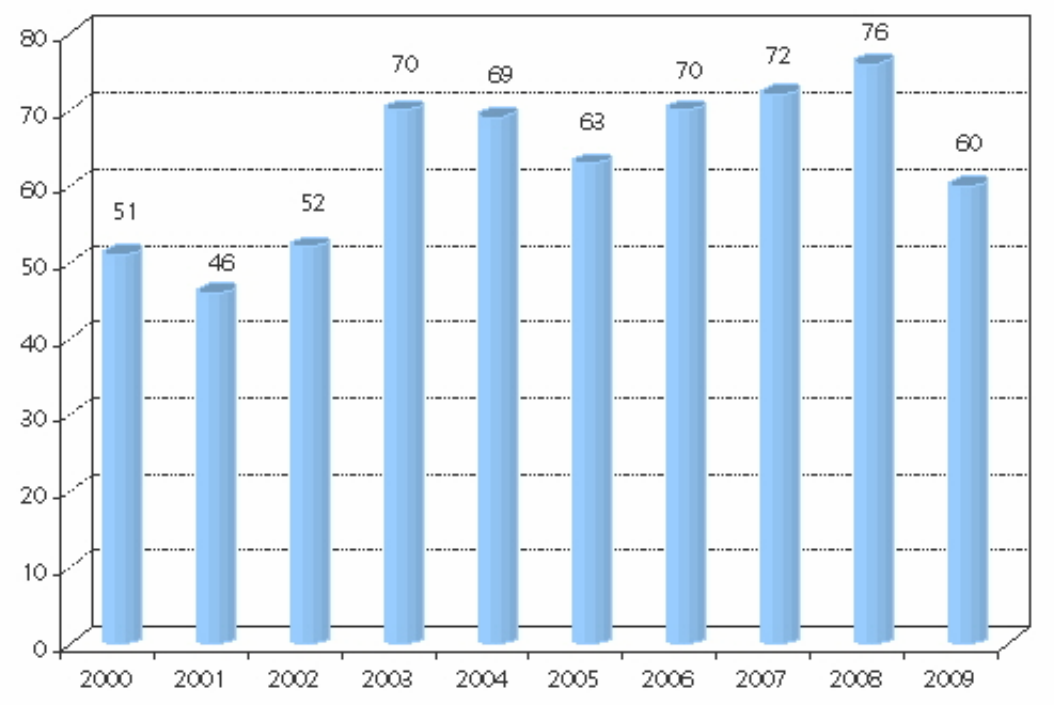

Los gráficos anteriores representan la evolución del número de feminicidios de género. Nos muestra una cifra media en torno a 70 casos por año con un pico máximo de 97 (año 1996) y mínimo de 46 (año 2001). Un examen superficial de esos datos podría llevar a la conclusión de que nada ha cambiado, dado que las cifras tras más de una década se mantienen a grandes rasgos estables. Sin embargo, dicha conclusión debe ser matizada introduciendo factores correctores $y$, entre ellos, creo que han de destacarse dos por encima de los demás:

En primer lugar, debe tenerse en cuenta la evolución del porcentaje de autores y víctimas del comportamiento violento analizado en función de su nacionalidad, pues parte de los esfuerzos de los últimos años se han centrado en medidas de tipo educativo y sobre todo de conformación de una conciencia social del ciudadano frente al maltrato (proceso que se prolonga durante más de una década). En efecto, una parte importante (aunque no la que más) de la política criminal adoptada frente a la violencia de género se ha basado en la concienciación social y cómo forma de repulsa al fenómeno, a la vez que se ha tratado de educar frente a los roles discriminatorios históricamente atribuidos al hombre y a la mujer, que han favorecido este fenómeno. Las políticas de intervención social se presentan como las más efectivas, especialmente aquellas que propician un 
cambio de comportamiento progresivo y el rechazo respecto a quienes mantienen tales actitudes. Sin embargo, la valoración de sus resultados requiere el transcurso de un lapso temporal elevado; es por ello, por lo que no siempre son las más rentables en términos político-electorales. Ese tipo de medidas y sus consecuencias no han llegado o al menos lo han hecho en menor medida a buena parte del colectivo de inmigrantes, que se han ido incorporando progresivamente a nuestra sociedad, provenientes además -con frecuencia- de ámbitos geográficos en los que se mantienen fuertemente arraigadas las pautas educativas y sociales que han facilitado las situaciones de violencia. Pues bien, observando el gráfico que aparece a continuación, comprobamos como hoy casi un tercio de los autores y víctimas de los feminicidios de género son extranjeros, mientras que cuando empezaron a recopilarse estos datos, la práctica totalidad eran nacionales.

[FIGURA 3A. Distribución por nacionalidad. Fuente gráfica: Centro Reina Sofía para el estudio de la violencia] $]^{3}$

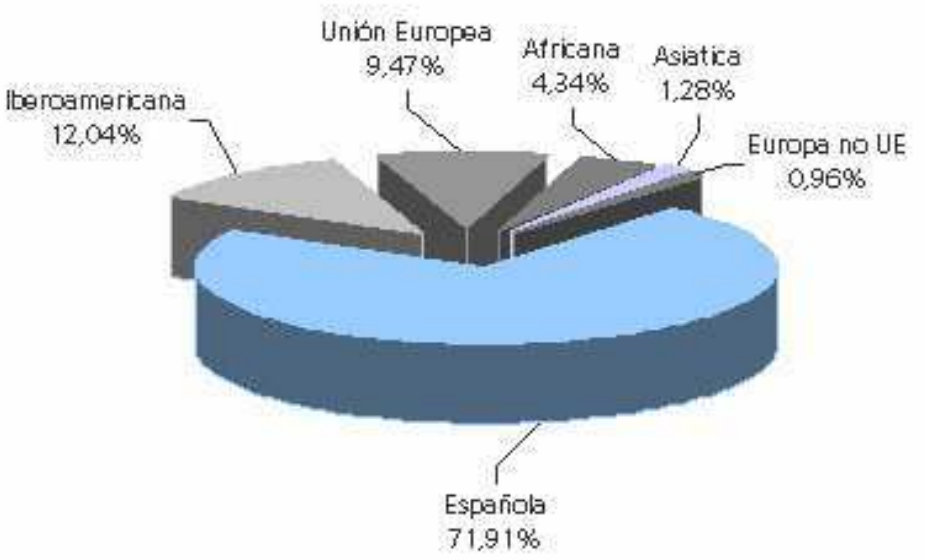

\footnotetext{
${ }^{3}$ Según el informe del año 2010 del CGPJ sobre violencia de género y en relación con la nacionalidad del autor de los feminicidios resulta que éste es español en 29 sentencias, equivalente al 72,5\% de supuestos. En el resto, un 27,5\% de casos -11 sentencias-, el autor es extranjero. Dichos porcentajes, a efectos de su valoración, deben relacionarse con la tasa de población española y extranjera (según datos del INE, referidos a 2.008, la población española ascendía a 46.157 .822 h., de los que 5.268.762 eran extranjeros, algo más del 11\%). En cuanto a la nacionalidad, de las resoluciones que recogen este dato, el $72 \%$ de las víctimas mortales son españolas, mientras que en un $28 \%$ son extranjeras.
} 
[FIGURA 3B. Evolución según la nacionalidad. Fuente gráfica: Centro Reina Sofía para el estudio de la violencia]

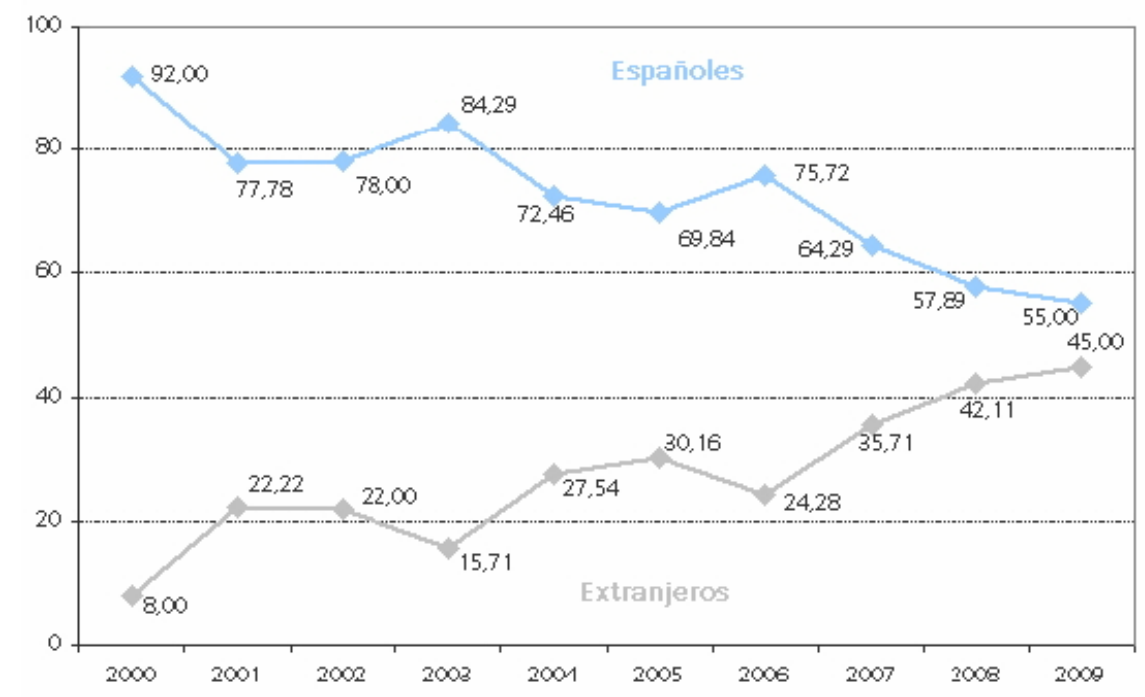

El segundo factor corrector debe relacionarse con el espectacular aumento del número de denuncias que se ha producido a lo largo de la última década y que aparece reflejado en los gráficos 3, 4 y 5. Pues bien, hay acuerdo en considerar que precisamente la ruptura de la relación (de dominio), a través de la denuncia del maltrato o el anuncio de separación o divorcio, es un factor de máximo riesgo para desencadenar situaciones de violencia y, en algunos casos, el feminicidio ${ }^{4}$. Ello nos permite deducir que la multiplicación exponencial del número de denuncias debería haber producido también un aumento significativo del número de víctimas mortales ya que, como se ha demostrado (con el análisis de las Órdenes de Protección adoptadas), la seguridad física absoluta de la víctima potencial no es viable ni en el nuestro ni en ningún otro modelo; sin embargo dicho aumento (en el número de feminicidios) no se ha producido, por lo que parece lógico pensar que el mismo se ha visto compensado negativamente por otros factores desactivadores.

La principal consecuencia que se obtiene al analizar un aumento tan significativo del número de denuncias (más de un $600 \%$ en el periodo gráficamente representado) no puede ser un equivalente aumento de las situaciones de maltrato, pues no hay razón que

\footnotetext{
${ }^{4}$ Ya advertía el Informe del Defensor del Pueblo de 1998 que nada menos que "el 98\% de las víctimas muertas habían presentado denuncia y estaban separadas o en trámite de separación del agresor".
} 
lo justifique, sino que, por el contrario, refleja la superación parcial de algunos de los grandes factores inhibidores que impedían que la denuncia se produjera (rechazo social, policial, judicial y sobre todo la desprotección de la víctima denunciante y el riesgo para su integridad física y condiciones socioeconómicas de futuro). Precisamente en el dato anterior (número de víctimas) encontramos el factor que avala esta afirmación, pues no parece discutible que las víctimas sean el vértice de una pirámide que descansa sobre una base sustentada sobre cientos de miles de casos de maltrato. Según este esquema y con carácter general, el aumento del número de casos de violencia de género debería desembocar, al menos a medio-largo plazo, en un aumento proporcional del número de víctimas mortales o feminicidios de género.

[FIGURA 4: DENUNCIAS (1994-1997). Fuente Gráfica: Informe del Defensor del Pueblo $(1998)]^{5}$

\begin{tabular}{|c|c|}
\hline AÑo & DENUNCIAS \\
\hline 1994 & 13.547 \\
\hline 1995 & 13.278 \\
\hline 1996 & 13.198 \\
\hline 1997 & 24.641 \\
\hline
\end{tabular}

[FIGURA 5 DENUNCIAS (2002-2006). Fuente gráfica: Ministerio del Interior]

\begin{tabular}{llllll}
\cline { 2 - 6 } & 2002 & 2003 & 2004 & 2005 & 2006 \\
\cline { 2 - 6 } & & & & & \\
\hline DELITOS & & & & & \\
\hline 11.047 & 15.462 & 40.518 & 49.237 & 533 \\
\hline
\end{tabular}

\footnotetext{
${ }^{5}$ En dicho informe ya se expresaba que "el número de denuncias de mujeres que han sido víctimas de malos tratos ha ido creciendo. El incremento experimentado desde 1984 no significa que haya aumentado el número de mujeres maltratadas, sino que la mujer ha tenido acceso a la información y ha ido conociendo los cambios legislativos, sus derechos y los recursos sociales que existen a su disposición".
} 
[FIGURA 6 DENUNCIAS. Suma de delitos y faltas (2006-2010): Fuente gráfica. Observatorio de la violencia de género]

\section{EVOLUCIÓN TRIMESTRAL DENUNCIAS}

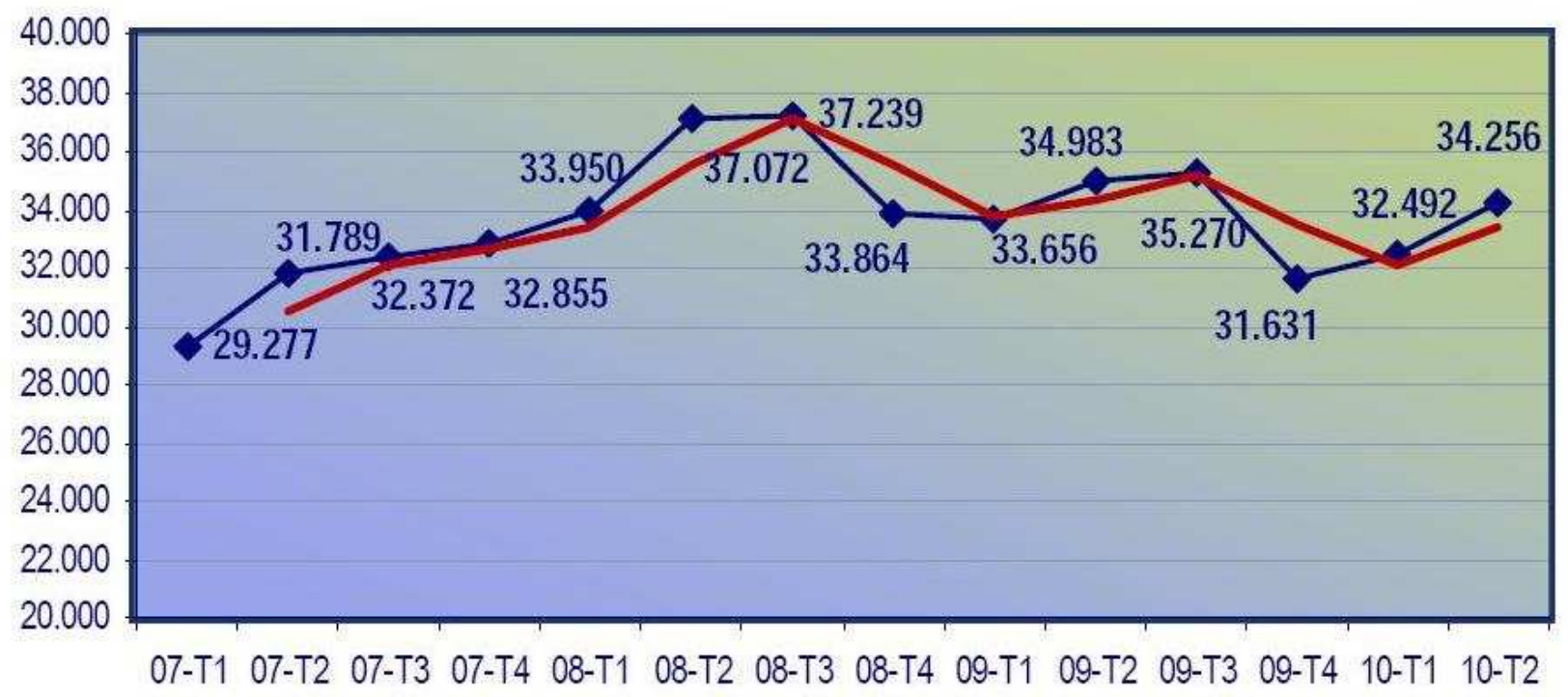

\section{Feminicidios de género seguidos de posterior suicidio del agresor (F/S)}

\subsection{Marco general y cuantificación}

El homicidio seguido de suicidio (homicidio/suicidio o H/S) se define técnicamente como la muerte violenta de otro seguida del suicidio del agresor dentro del plazo de 24 horas (Van Wormer, 2008), si bien en la práctica ambos sucesos suelen ser consecutivos sin mediar apenas espacio temporal entre ellos. Dentro de esa categoría genérica se incluye el fenómeno de los feminicidios de pareja seguidos del suicidio del agresor $(\mathrm{F} / \mathrm{S})$; de hecho, es ese el supuesto de $\mathrm{H} / \mathrm{S}$, que se produce con más frecuencia; en concreto, en algunos estudios realizados (Logan et. Al. 2008), se comprobó cómo los F/S constituyen en Estados Unidos el 74,5\% del total de los casos de $\mathrm{H} / \mathrm{S}$. Nos encontramos, por otra parte, ante un tipo de reacción (suicidio tentado o consumado tras dar muerte a la pareja) que se aleja de modo radical de lo característico, no sólo en el resto de delincuencia, sino también en la delincuencia violenta extrema (aquella que tiene resultados mortales). La primera reflexión que puede hacerse es que lo anterior no viene más que a ratificar la extraordinaria singularidad de este tipo de crímenes 
violentos y la consiguiente necesidad de establecer pautas de actuación y tratamiento claramente diferenciadas respecto a las utilizadas frente al resto de la criminalidad violenta.

El suicidio (consumado o tentado) después de la comisión de un delito grave, entre ellos el homicidio o feminicidio dolosos, es un hecho excepcional; un fenómeno extraño y que raras veces acontece. Sin embargo, esos porcentajes -ínfimos desde un punto de vista estadístico- se ven alterados de un modo radical cuando se trata de feminicidios de género, en cuyo ámbito se produce un aumento espectacular. En Estados Unidos algunos estudios determinaron que esta cifra era superior al 40\% (Lund L. E., Smorodinsky S., 2001); otros trabajos realizados con datos de Chicago y Carolina del Norte concluyeron que en todo caso representa una proporción significativa (entre el $27 \%$ y el $32 \%$ ), que sin embargo se convierte en algo absolutamente marginal cuando es la mujer la que mata al hombre (0,1\%) (Campbell et al. 2007). En España, el porcentaje de suicidios (tentados o consumados) se sitúa en los últimos años en porcentajes superiores al 30\%; puede decirse que uno de cada tres feminicidas de género se suicida o al menos lo intenta. Debe añadirse además que las estadísticas no recogen a aquellos que realmente manejaron ideas suicidas pero no han sabido cómo gestionarlas.

[FIGURA 7. SUICIDIO DE FEMINICIDAS. Fuente Gráfica: Ministerio de Igualdad]

Año 2006

\begin{tabular}{|c|l|r|r|}
\hline \multirow{2}{*}{$\begin{array}{c}\text { SUICIDIO DEL } \\
\text { AGRESOR }\end{array}$} & No & 43 & $62,3 \%$ \\
\cline { 2 - 4 } & Tentativa & 9 & $13,0 \%$ \\
\cline { 2 - 4 } & Suicidio consumado & 17 & $24,6 \%$ \\
\hline
\end{tabular}

Año 2007

\begin{tabular}{|c|l|r|r|}
\hline \multirow{2}{*}{$\begin{array}{c}\text { SUICIDIO DEL } \\
\text { AGRESOR }\end{array}$} & No & 56 & $78,9 \%$ \\
\cline { 2 - 4 } & Tentativa & 10 & $14,1 \%$ \\
\cline { 2 - 4 } & Suicidio consumado & 5 & $7,0 \%$ \\
\hline
\end{tabular}

Año 2008

\begin{tabular}{|c|l|r|r|}
\hline \multirow{2}{*}{$\begin{array}{c}\text { SUICIDIO DEL } \\
\text { AGRESOR }\end{array}$} & No & 53 & $69,7 \%$ \\
\cline { 2 - 4 } & Tentativa & 7 & $9,2 \%$ \\
\cline { 2 - 4 } & Suicidio consumado & 16 & $21,1 \%$ \\
\hline
\end{tabular}


Año 2009

\begin{tabular}{|c|l|r|r|}
\hline \multirow{2}{*}{$\begin{array}{c}\text { SUICIDIO DEL } \\
\text { AGRESOR }\end{array}$} & No & 35 & $62,5 \%$ \\
\cline { 2 - 4 } & Tentativa & 8 & $14,3 \%$ \\
\cline { 2 - 4 } & Suicidio consumado & 13 & $23,2 \%$ \\
\hline
\end{tabular}

En general, y frente al resto de muertes violentas no hay apenas reacción de autoprotección del agresor frente al sistema (policial, judicial y penitenciario) tras cometer su crimen; normalmente lejos de huir, manifiesta reacciones de sumisión frente a dicho sistema; se entrega o espera a ser detenido (con frecuencia el mismo da noticia de su crimen a las autoridades) o, como vemos, procura su propia eliminación física. Es un sujeto que resuelve de modo violento un conflicto, frente al que no encuentra alternativas, lo percibe de un modo extraordinariamente intenso y o bien procura su propia eliminación o no muestra oposición a las consecuencias muy negativas previstas por el sistema para su comportamiento ${ }^{6}$, pese a que no las comparte, pues normalmente está plenamente convencido de lo correcto y necesario de su actuación. Es por lo tanto, en este aspecto, un sujeto escasa o nulamente motivable. En efecto, el estado anímico característico del momento de la ejecución de los hechos contribuye también a desactivar parcialmente la efectividad de los instrumentos de intimidación y control, en particular en el caso de existir una medida de las contenidas en el art. $48 \mathrm{CP}$, dirigidas a evitar el contacto entre el agresor y la posible víctima de un feminicidio.

\subsection{Perfiles y predictores del modelo de feminicidio-suicidio}

Con una base teórica psicodinámica, Meenninger ya en 1970 exponía los tres deseos que podrían llevar al acto suicida: el deseo de morir, el deseo de matar y el deseo de estar muerto (Dubugras B., Guevara W., 2007). Lo más relevante y principal peculiaridad del fenómeno, en todo caso, tal y como constatan varios estudios, es que no se trata de una decisión de homicidio que da lugar a una (posterior) decisión de suicidio, como dos hechos distintos; por el contrario ambas conductas homicidio y posterior

\footnotetext{
${ }^{6}$ Según el análisis de sentencias referidas al año 2009 realizado por el Consejo General del Poder Judicial, y en lo que respecta a la conducta del acusado tras los hechos, la mayor parte de las 34 resoluciones analizadas - un $85 \%$ de la muestra- refieren la entrega voluntaria del autor.
} 
suicidio $(\mathrm{H} / \mathrm{S})$ obedecen a un plan común y generalmente ambos están bien planificados como unidad y apenas se aprecian rasgos de improvisación (Dubugras B., Guevara W., 2007). Entre otras conclusiones, ello nos debiera permitir trazar una línea diferenciadora respecto a los supuestos de homicidio en los que está ausente el ánimo suicida, siendo la situación anímica previa completamente diferente a la de aquellos homicidios que se producen como consecuencia de un estallido de tensión, los cuales -por el contrarionormalmente no van acompañados del suicidio del agresor. En efecto, los perfiles del que (únicamente) mata y del que mata y (además) se suicida son distintos (Lund L. E., Smorodinsky S., 2001). Con carácter general, el perfil del homicida-suicida curiosamente se aproxima bastante más al perfil del suicida que al del homicida (Dubugras B., Guevara W., 2007). También es distinto el del que mata y se suicida del que mata por razones de género y después se suicida.

MiLROY (1998) encontró que el suicidio tras el homicidio es parte de un caso de "suicidio ampliado", y por lo tanto diferente a otros tipos de homicidios. La mayoría de estudios apuntan la presencia en el $\mathrm{H} / \mathrm{S}$ de las características demográficas de las personas que se suicidan más que las de los que cometen homicidio (Kachur, et al., 1995). En todo caso, hay opiniones encontradas sobre este aspecto (Voigt L., 2007) ${ }^{7}$.

Es característica de todo $\mathrm{H} / \mathrm{S}$, la existencia de un nivel de dominación y dependencia entre el autor y la víctima, con lo cual parece necesario incorporar también a este modelo -como antes se apuntó- la idea del cuestionamiento o ruptura de esa relación de control como causa de ambos comportamientos que conformarían una unidad (Voigt L., 2007). Observamos que precisamente es esta una peculiaridad presente de forma muy intensa en los Feminicidios-Suicidios $(\mathrm{F} / \mathrm{S})$ de género. El deterioro y grave alteración psicológica que produce al agresor la ruptura de su esquema vital de dominio puede coincidir parcialmente con el estado mental y emocional de algunos suicidas.

\footnotetext{
${ }^{7}$ Para unos es una subclase de homicidio (por ejemplo, Palermo, 1994; Palmer \& Stack, 1997; Wallace, 1986); para otros es una subclase de suicidio (por ejemplo, Marzuk y Humphrey, 1980; West, 1966; Wolfgang, 1958). Otros opinan que son dos eventos distintos (por ejemplo, Marzuk et al., 1992).
} 
Otras características aparecen también en los estudios citados, algunos de los cuales concluyen que, en general, suele tratarse de personas que fuera de ese contexto, pocas veces se comportarían con dosis de violencia elevadas capaces de causar a la muerte a otro, siendo ésta otra peculiaridad que los diferencia parcialmente del feminicida no-suicida.

En cuanto a concretas características demográficas del F/S, se observa (Lund L. E., Smorodinsky S., 2001) que la media de edad de los agresores que se quitan la vida es superior a la media de los feminicidas en los que no concurren actos suicidas; parece que ello no viene más que a reafirmar las explicaciones bajo el modelo expuesto, en la medida en que la relación de dominio se afianza con los años y, con muchos matices, ha sido más propia de otro momento histórico vivido por personas que ahora se encuentran en estratos de edad superiores. En España, haciendo el cálculo utilizando los datos referidos a la edad del agresor, proporcionados por el Centro Reina Sofía para el estudio de la violencia, podemos observar que la edad media del agresor suicida (con datos del año 2010) es de 54,5 años, casi 7 superior a la media general del feminicida $(47,7$ años) y casi 10 a la media del feminicida no suicida (45 años).

También se ha observado que el uso de armas y en particular de un arma de fuego es un indicador de un posible suicidio tras el feminicidio. Los trabajos desarrollados y conclusiones obtenidas por la doctrina estadounidense, en las que el uso de arma de fuego está presente de modo muy mayoritario en los supuestos de F/S (Campbell J. C., Webster D., et al., 2003 y Logan J., Hill H. A. Black M. L., Crosby A. E., Karch D. L, Barnes J. D., Lubell K. M., 2008), no son del todo extrapolables a nuestro país, por la conocida profusión de este tipo de armas en Estados Unidos. Sin embargo, si analizamos la información datos del Centro Reina Sofía para el estudio de la violencia (año 2010), comprobamos que en los supuestos en que el agresor usa armas $\mathrm{y}$, en particular armas de fuego, aumenta de modo significativo la probabilidad de suicidios (tentados o consumados). Así, de los 74 feminicidios de género acaecidos en el año 2010, el suicidio o intento del mismo consta en 23 casos, lo que constituye un $31 \%$ del total. En 54 ocasiones el agresor utilizó armas (69\%) y en 8 estas eran de fuego (10,8\%). Frente a ello, en 18 de los 23 casos el agresor suicida, utilizó armas (78\%, 9 
puntos más), siendo estas de fuego en 6 ocasiones, (26\%) lo que constituye un incremento de más de 15 puntos porcentuales.

\subsection{Causas del suicidio posterior al homicidio}

Desde hace tiempo se viene defendiendo una teoría, según la cual, la muerte violenta de la mujer y el posterior suicidio del agresor se explicaría de acuerdo a la dificultad del agresor de soportar el fuerte rechazo social que su acción comporta. Sin embargo, no parece ser esa la hipótesis correcta; el conocimiento actual nos permite comprobar cómo el agresor de género que termina con la vida de mujer es escasamente permeable a los estímulos externos; así se comprueba por ejemplo con las experiencias obtenidas con los tratamientos desarrollados en centros penitenciarios con los agresores de género; en ellos se pone de manifiesto que el tipo más frecuente de agresor es un sujeto victimista absolutamente convencido de lo correcto de su actuación y de lo "merecido" del castigo infligido a su pareja por su desviación de las pautas y obligaciones que para con él están establecidas. Con frecuencia, precisamente la no superación de ese esquema impide el éxito del tratamiento.

Una parte importante de los agresores violentos de género que llegan al feminicidio han conformado su modelo o esquema vital sobre la base de una relación de dominio y control absoluto de su pareja, en la que utilizan la violencia para reafirmar el dominio y, si fuera necesario, restablecer el control. La pérdida brusca de ese modelo vital (que consideraban blindado) termina con la "necesaria" eliminación física de la mujer, proceso que a veces determina la ampliación a la propia muerte del agresor (feminicidio ampliado), al entender carente de sentido su existencia sin ese modelo de dominio en torno al que gravita la misma.

En el fondo, la idea sobre la que se sustenta el F/S es la de la solución dramática de la ruptura no asumida por el varón, dado el modelo deformado sobre el que ha construido su relación y su pleno convencimiento de que es el correcto y único posible. La principal diferencia entre el feminicidio no suicida y el F/S radicaría entonces en el mayor o menor arraigo de ese modelo, en cuyo caso se plantea como un conflicto radical no solucionable, siquiera con la muerte violenta de la pareja o expareja. Por ello, 
en este tipo de situaciones resultan decisivos los estímulos de ruptura (anuncio de separación, divorcio o denuncia por maltrato). En efecto, la ruptura (el divorcio o separación) o la amenaza creíble de la misma, parece ser el principal factor de riesgo del feminicidio y en particular del F/S, cuando el modelo y por lo tanto el conflicto está profundamente arraigado (Logan J., Hill H. A. Black M. L., Crosby A. E., Karch D. L, Barnes J. D., Lubell K. M., 2008). Según los estudios existentes sobre la materia, el riesgo de feminicidio de pareja se incrementa 9 veces si a la relación basada en el abuso físico y emocional se le añade una separación o amenaza de la misma por parte de la mujer Campbell J. C., Webster D., Koziol-McLain J., Block C. et alt., 2003.

Con datos concretos, ha llegado a las mismas conclusiones el CGPJ en su análisis de sentencias relativas a feminicidios de género ${ }^{8}$ : "Estos datos continúan confirmando la apreciación, constatada en los anteriores estudios: la advertencia o comunicación del deseo de separase por parte de la mujer o la propia materialización de la ruptura constituyen un específico factor de riesgo, en cuanto detonante de la reacción extrema del agresor. Como se concluía en estudios anteriores, tales datos siguen reflejando que, más que los conflictos puntuales surgidos de la relación de convivencia, es el propio modelo de relación establecido, asimétrico en las relaciones de poder, el que produce estos resultados criminales.

En definitiva, resulta imprescindible el control de las situaciones de riesgo activadas por la ruptura del vínculo o la manifestación por parte de la mujer del deseo de llevarla a cabo. En tal sentido, deben establecerse fórmulas de protección ante tal decisión en situaciones de riesgo que deben ser autoevaluadas por la propia víctima potencial, evitando -a partir de ese momento- la comunicación directa con el agresor. Resulta llamativo como, estando identificado como uno de los principales factores de riesgo, no existan sin embargo instrumentos específicos de protección o autoprotección. Recordemos, además, que es precisamente en estos casos donde se produce un mayor

\footnotetext{
${ }^{8}$ Análisis de las sentencias dictadas por los tribunales del jurado y por las audiencias provinciales en el año 2008, relativas a homicidios y/o feminicidios consumados entre los miembros de la pareja o ex pareja (Consejo General del Poder Judicial); "al menos en un caso, se declara probada la previa comunicación de la esposa de su intención de separarse, en otro la separación se había producido recientemente, en otro la separación había tenido lugar con anterioridad y hay 4 sentencias que declaran probada la previa incoación de un proceso civil de separación o divorcio. En un 37\% -14 de las 38 sentencias que recogen el dato- la relación de afectividad no se mantenía".
} 
porcentaje de suicidios (consumados o tentados), lo que en definitiva pone de manifiesto que la motivación de la amenaza penal es prácticamente nula y requiere de otros factores inhibidores de la violencia extrema absoluta.

\section{EI denominado "efecto llamada" en los feminicidios de género. Estudio de la aleatoriedad en la distribución temporal de los feminicidios.}

\subsection{Aspectos previos: Planteamiento, referentes y nomen utilizado}

Nos ocupamos a continuación de la cuantificación y análisis criminológico de diversas variables y en particular del estudio de una hipótesis ampliamente difundida por los medios de comunicación españoles ${ }^{9}$ y por diversos responsables políticos con competencias en la lucha contra la violencia de género. Según la misma, la difusión por parte de los medios de noticias de violencia de género extrema (feminicidios o muertes violentas de las mujeres por parte de sus parejas o ex parejas) estimularía la realización del mismo comportamiento en otros sujetos que se encuentran en situaciones de riesgo (de llevar a cabo tales comportamientos); expresado de modo más directo: matar al conocer que otros han matado. Según esta hipótesis, tales sujetos se verían reforzados desde un punto de vista moral o recibirían un estímulo necesario y final para llevar a cabo el comportamiento de violencia extrema, terminando con la vida de la mujer. También desde un punto de vista doctrinal, se ha afirmado la existencia de este "efecto llamada” o “imitación” (Vives-Cases, C. Torrubiano-Domínguez J., Álvarez-Dardet C., 2009). En el trabajo citado se analiza la profusión de noticias sobre la materia y sus autores comprueban la existencia de diferencias significativas los días anteriores y

\footnotetext{
${ }^{9}$ http://www.nortecastilla.es/v/20101027/segovia/lorente-afirma-menos-victimas-20101027.html http://www.elpais.com/articulo/sociedad/Mueren/mujeres/feminicidios/machistas/meses/todo/2009/elpep usoc/20101015elpepusoc_1/Tes http://www.elpais.com/articulo/sociedad/efecto/llamada/malos/tratos/elpepusoc/20070621elpepisoc 2/Te $\underline{\mathrm{S}}$ http://www.elconfidencial.com/cache/2007/10/28/33 crtve_seguira_informando_sobre_casos_violencia genero.html

http://www.levante-emv.com/opinion/2008/07/08/opinion-violencia-genero-prensa/469696.html http://www.elpais.com/articulo/sociedad/falaz/efecto/llamada/maltrato/elpepusoc/20070714elpepisoc 4/T es

http://www.ideal.es/jaen/prensa/20070722/local_jaen/mayoria-homicidios-violenciagenero_20070722.html entre muchos otros [Consulta 28-01-2011].
} 
posteriores a un feminicidio, pero utilizando para ello un periodo determinado, en concreto la comparación entre los 7 días anteriores y posteriores a la comisión de un homicidio de género ${ }^{10}$.

Al fenómeno descrito se le ha venido denominado de diferentes maneras: “efecto llamada", "efecto imitación" e incluso "efecto repetición”. Si partimos de que se trata de un sujeto que se encuentra en situación potencial de comportarse como autor de un ataque violento extremo de género, no parece del todo correcto hablar de "llamada" (el comportamiento de unos estimula la acción de otros en el mismo sentido). Tampoco parece totalmente adecuada la expresión "efecto imitación"; no es que se copie algo que hacen otros, sino que el conocimiento de que otros, probablemente en un estado personal y emocional semejante han llevado a cabo un comportamiento de este tipo reforzaría moralmente, estimularía o serviría como ingrediente final a un proceso que normalmente viene configurándose desde mucho tiempo antes. Podría optarse en consecuencia por denominarlo "efecto estímulo, estimulador o desencadenante" de la violencia. El conocimiento del comportamiento violento de otros, bajo condiciones muy determinadas, podría servir como estímulo o elemento desencadenante de un proceso que se encuentra en su fase final o de máximo riesgo. Utilizamos en todo caso en este trabajo la expresión "efecto llamada", al ser la más difundida hasta la fecha.

Nos preguntamos, en definitiva, si puede contribuir la información reflejada en los medios sobre crímenes de género a activar en el maltratador el comportamiento de violencia extrema equivalente, ¿realmente produce un reforzamiento moral a aquellos que se encuentran en una situación de riesgo potencial de ser autores de un comportamiento violento capaz de terminar con la vida de la mujer? Hoy sabemos que un comportamiento criminal o delictivo violento se activa en la generalidad de los casos no por un factor individual, sino por la suma o combinación de múltiples de ellos; en el caso objeto de estudio, dicha concurrencia de factores incrementa las situaciones de riesgo y la aparición de un elemento más (el supuesto efecto estimulante derivado del

\footnotetext{
${ }^{10}$ Relacionan este fenómeno con otros como los homicidios y los suicidios. Respecto a los primeros concluyen que es difícil hacer un estudio riguroso y establecer una separación a efectos estadísticos, al ser un evento que se producen de forma continua y reiterada, al margen de comportamientos homicidas. Por lo que respecta al suicidio, nos recuerdan como está plenamente constatado como influye en la decisión (aumentando las posibilidades de suicidio) de quien conoce o tiene noticia de que otro/s lo han hecho (se han suicidado).
} 
conocimiento de que otros, en una situación parecida, han acabado con la vida de su pareja) podría desencadenar -como estímulo final- el comportamiento feminicida. Lo anterior se vería potenciado por la aparente escasa capacidad de motivación a los agresores de género a través de la amenaza penal (Saridakis G, 2004). Por lo tanto, no debe descartarse a priori como hipótesis una posible relevancia práctica de este factor, como elemento de reforzamiento moral, que puede sufrir quien conoce que otro ha sido capaz de llevar a cabo el comportamiento que el mismo - de modo más o menos consciente- viene manejando como posibilidad.

Y, en efecto, una observación del calendario, puede sugerir cierta agrupación temporal de feminicidios (Figura 8). Por ello vamos a analizar si dicha distribución sigue algún tipo de racha o patrón temporal o por el contrario se puede considerar que tiene lugar una distribución aleatoria uniforme.

[FIGURA 8. Distribución temporal de los 61 feminicidios producidos durante el año 2009. Elaboración propia]

$$
\mathrm{X} \text { Un asesinato; } \mathbf{x} \text { Dos asesinatos }
$$

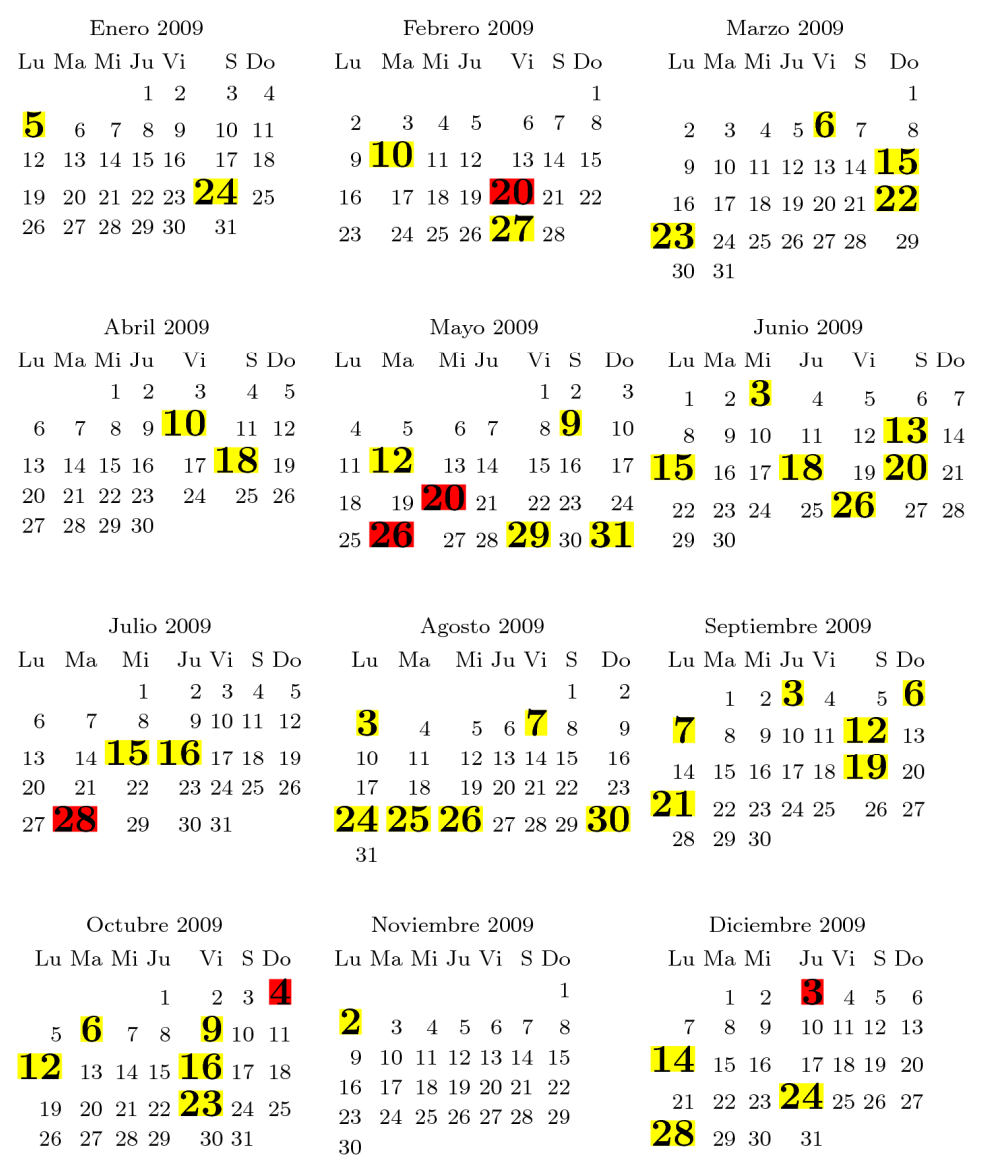




\section{Resultados}

\section{Frecuencia de feminicidios}

Se produjeron 346 feminicidios comprendidos entre enero de 2005 y abril de 2010, un periodo que abarca 1946 días. El número medio de feminicidios por día es 0.178 , lo que representa de media un feminicidio cada 5.62 días y un promedio anual de 64.89. Los días en los que hubo feminicidios fueron 296 (el 15.7\% del total), de los cuales en 36 y en 2 días hubo dos y tres, respectivamente (Figura 9).

[FIGURA 9: Días en los que hubo feminicidios y el número de coincidencias. Elaboración propia]

\begin{tabular}{lrrrr}
$\mathbf{N}^{\mathbf{0}}$ feminicidios & \multicolumn{1}{c}{$\mathbf{N}^{\mathbf{0}}$ días } & \% & \multicolumn{2}{c}{ Acum. \% } \\
0 & 1640 & 84.3 & 84.3 \\
1 & 268 & 13.8 & 98.0 \\
2 & 36 & 1.8 & 99.9 \\
3 & 2 & 0.1 & 100.0 \\
\hline Total & 1946 & 100.0 & 100.0
\end{tabular}

\section{Distribución mensual}

Agrupando los feminicidios según el mes del año en el que ocurren, el número medio varía desde 4.2 casos de media en noviembre hasta los 7.0 feminicidios de media en agosto. Dado que la serie histórica abarca menos de 6 años, no se detectan diferencias significativas en el número de asesinadas por mes (Kruskall-Wallis de rangos $_{\text {g.l.11 }}=10.16, \quad \mathrm{p}$-valor $=0.5163$ ) ni presencia de estacionalidad (tanto la autocorrelación como la parcial son no significativas en los retardos) (Figuras 10 y 11). Debe advertirse, sin embargo, que parece lógico pensar que una buena parte de los feminicidios que se producen los lunes, tienen lugar en la madrugada del domingo al lunes, por lo que materialmente también se podrían considerar cometidos durante el fin de semana. 
[FIGURAS 10. Número medio de feminicidios según el mes del año. Período Enero de 2005 a Abril de 2010. Elaboración propia]

\begin{tabular}{rl} 
Número medio de asesinatos según el mes del año. \\
Período Enero de 2005 a Abril de 2010. \\
\hline Mes & Número medio de asesinadas \\
\hline 1 & 5.17 \\
2 & 6.17 \\
3 & 4.67 \\
4 & 4.33 \\
5 & 5.4 \\
6 & 6 \\
7 & 6.8 \\
8 & 7 \\
9 & 4.6 \\
10 & 5.6 \\
11 & 4.2 \\
12 & 5.2 \\
\hline
\end{tabular}

[FIGURA 11. Distribución temporal del número de asesinadas, agrupada por meses]

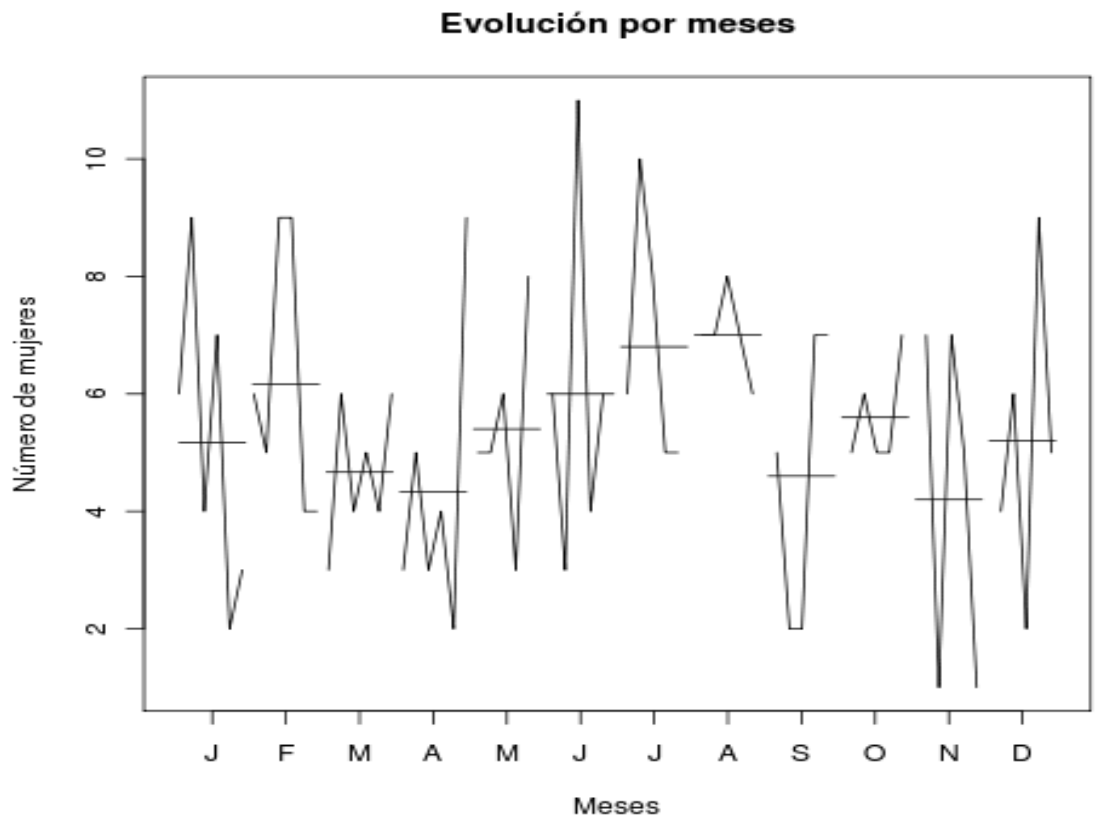




\section{Distribución semanal}

La distribución semanal de los feminicidios presenta su máximo en el domingo (17.6\%) seguido del lunes (16.8\%). Sin embargo, las variaciones observadas no son muy significativas respecto a la distribución semanal, $\mathrm{p}$-valor=0.289).

[FIGURA 12. Distribución semanal de los feminicidios]

\begin{tabular}{lccc} 
& Frec. & \% & \multicolumn{2}{c}{ Acum. \% } \\
Lunes* & 58 & $\mathbf{1 6 . 8}$ & 16.8 \\
Martes & 50 & 14.5 & 31.2 \\
miércoles & 42 & 12.1 & 43.4 \\
Jueves & 50 & 14.5 & 57.8 \\
Viernes & 41 & 11.8 & 69.7 \\
Sábado & 44 & 12.7 & 82.4 \\
domingo & 61 & $\mathbf{1 7 . 6}$ & 100.0 \\
\hline Total & 346 & 100.0 & 100.0
\end{tabular}

[FIGURA 13. Distribución temporal del número de víctimas mortales, agrupada por días de la semana]

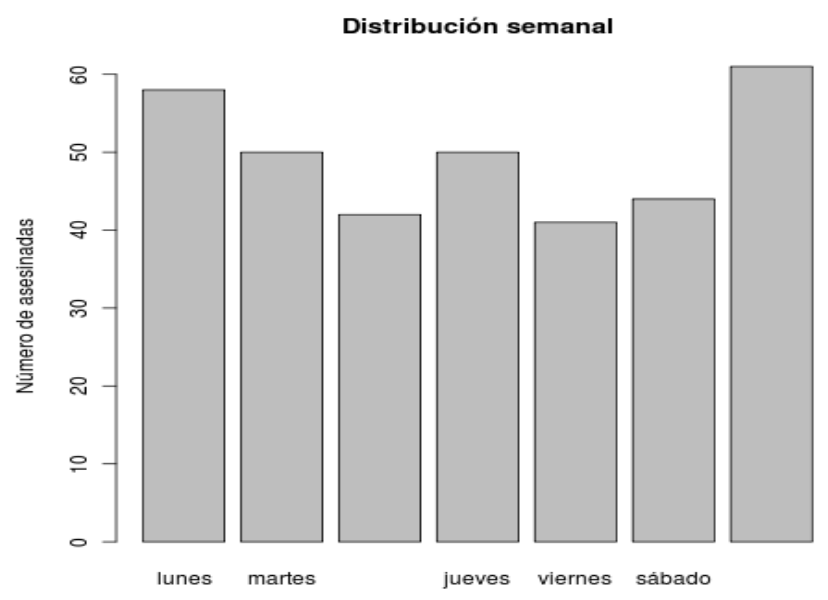


Lapso en días entre feminicidios consecutivos

Calculando el lapso diario entre feminicidios consecutivos, se observa que el tiempo medio de demora entre feminicidios es de 5.61 días, con desviación típica $5.97 \mathrm{y}$ mediana 4.00 días. El valor mínimo y máximo de retardo se alcanzan en 0.00 y 34.00 días, respectivamente. El 11.6\% de los casos acontecen el mismo día, el 12.5\% al día siguiente, el $37.4 \%$ de los mismos ocurren con dos o menos días de diferencia, el 56.5\% de feminicidios consecutivos ocurren con un lapso de 4 días o menos días y sólo el 17.4\% suceden después de 9 días del feminicidio precedente (figura 14).

[FIGURA 14. Lapso diario entre feminicidios consecutivos]

\begin{tabular}{lccc} 
Lapso (días) & Frec. & $\mathbf{\%}$ & \multicolumn{2}{c}{ Acum. \% } \\
0 & 40 & 11.6 & 11.6 \\
1 & 43 & 12.5 & 24.1 \\
2 & 46 & 13.3 & 37.4 \\
3 & 36 & 10.4 & 47.8 \\
4 & 30 & 8.7 & 56.5 \\
5 & 18 & 5.2 & 61.7 \\
6 & 25 & 7.2 & 69.0 \\
7 & 16 & 4.6 & 73.6 \\
8 & 16 & 4.6 & 78.3 \\
9 & 15 & 4.3 & 82.6 \\
$>9$ días & 60 & 17.4 & 100.0 \\
\hline Total & & 100.0 & 100.0
\end{tabular}

Distribución aleatoria de los sucesos

El periodo observado abarca desde el día 1 de enero de 2005 (día $1^{\circ}$ ) hasta el día 30 de abril de 2010 (día $1946^{\circ}$ ). En ese intervalo de tiempo acontecieron sucesos en los días $9^{\circ}, 12^{\circ}, 15^{\circ}, \ldots, 1930^{\circ}, 1936^{\circ}$ y $1945^{\circ}$. Contrastando esta distribución de días con una distribución aleatoria uniforme, se comprueba que la ocurrencia de feminicidios sigue dicha distribución aleatoria ( $\mathrm{p}$-valor=0.9597). 


\section{Relación temporal de los sucesos}

El análisis de los datos diarios de fallecimientos en el periodo considerado mediante la función de autocorrelación y función de autocorrelación parcial muestra indicios de relación temporal en algunos retardos (Fig. 15).

[FIGURA 15. Autocorrelación parcial de la serie diaria de feminicidios]

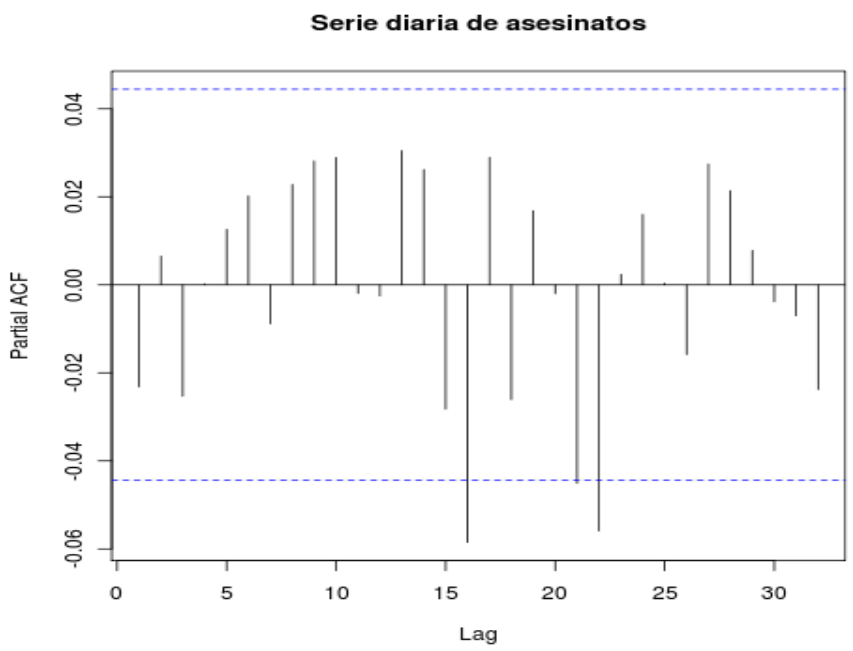

Dado que es un variable de tipo conteo con valores $0,1,2$ y 3 , los intervalos mostrados en la figura no son muy fiables. Se calculó una nueva variable dicotómica 0-1 indicando la presencia o no de feminicidios en un determinado día. La relación de esta variable con los retardos de 1 a 30 sólo aporta relación significativa en los retardos 13, 16 y 22 (Fig. 16).

[FIGURA 16. Coeficientes del modelo de regresión]

\begin{tabular}{lcccc} 
& Coeficiente & Desv. típica & Valor & \multicolumn{2}{c}{$\operatorname{Pr}(>|\mathbf{z}|)$} \\
$($ Constante $)$ & -1.6312 & 0.0772 & -21.13 & 0.0000 \\
retardo13 & 0.3461 & 0.1606 & 2.15 & 0.0312 \\
retardo16 & -0.4104 & 0.1921 & -2.14 & 0.0327 \\
retardo22 & -0.3744 & 0.1904 & -1.97 & 0.0492
\end{tabular}




\section{Conclusiones}

- La afirmación relativa a la ineficacia de las medidas contra la violencia de género en su versión más extrema (feminicidios) debe ser puesta en entredicho; si bien las cifras se mantienen estables, el proceso de evolución natural hubiera implicado un incremento muy significativo en el número de casos, al fomentarse de modo adecuado la ruptura de la relación de maltrato, siendo éste uno de los principales factores estimuladores del feminicidio. Del mismo modo, comprobamos que en la actualidad un porcentaje significativo de los autores (y de la víctimas) son extranjeros, circunstancia prácticamente inexistente hace una década, elemento que también debe ser valorado a la hora de analizar la mayor o menor eficacia de las políticas frente a la violencia de género y del rechazo de la sociedad a la misma, en la medida en que los ciudadanos extranjeros, por diferentes razones, no las han podido interiorizar del mismo modo que los nacionales.

- En lo que respecta al suicidio (consumado o tentado) del agresor tras la comisión del feminicidio -que hoy ya supera más de un tercio de los casos- nos permite poner de manifiesto de la cada vez mayor falta de motivación (inasequibilidad) de los agresores de género a la amenaza (intimidación) penal y sus consecuencias. Se han localizado predictores de este tipo de comportamientos como la mayor edad del agresor, la presencia de armas en el lugar de la agresión y el mayor arraigo del modelo de dominio del varón y sumisión de la mujer.

- La distribución temporal de feminicidios sigue un patrón aleatorio, de tal forma que se produce un suceso violento cada 5.62 días de media. Con los datos disponibles, no se sustenta estadísticamente la presencia de factores espurios en la distribución temporal de los mismos. La presencia de noticias en los medios de comunicación no influye en el número de feminicidios. En definitiva, no existe el denominado efecto llamada o estimulación. La contradicción de esta conclusión con el estudio antes citado creemos que se debe a que, en aquél apenas se hace énfasis en la distribución temporal de los feminicidios ya que, tal como señalan los autores, y respecto a la duración del periodo de comparación, se han utilizado menos días de comparación (referencias 17,20) e indican la distribución semanal de los feminicidios para justificar un periodo tan extenso. 
- Relación temporal de los sucesos. Existe relación entre lo que acontece 13, 16 y 22 días antes y que haya feminicidios un día concreto. Estadísticamente, que haya un feminicidio 13 días antes aumentaría la probabilidad de que se produzca otro, mientras que si lo hubo 16 o 22 días antes, disminuye la probabilidad de feminicidio. El resto de los días no influye. Evidentemente, al no haber ningún factor conocido que pueda justificar tales relaciones debe atribuirse a la pura casualidad y con la acumulación de datos de los próximos años debería ir disipándose.

- No se observa estacionalidad mensual en los feminicidios; la pequeña variación que, examinando los datos publicados, en este caso sí puede apreciarse, no tiene relevancia desde un punto de vista estadístico, al estar ausentes los márgenes de confianza necesarios. Este aspecto es muy relevante porque siempre se ha defendido la existencia de un notable incremento en los meses de verano, justificado por el mayor grado de convivencia entre los cónyuges y en menor medida el calor estival; vemos, sin embargo, como este factor con el análisis estadístico se diluye de forma notable.

- Tampoco existe una relación estadística significativa entre el día de la semana y el número de feminicidios (las variaciones observadas no son significativas respecto a la distribución semanal chi- -valor $=0.289$ y el periodograma de la serie diaria de feminicidios no indica ningún componente estacional semanal), y por otra parte, no se rechaza la distribución uniforme de un feminicidio cada 5.62 días y un promedio anual de 64.89 fallecimientos, independientemente de la presencia o no de noticias en los medios de comunicación ( $\mathrm{p}$-valor=0.9597). 


\section{Referencias}

Campbell J. C., Glass N., Sharps P. W., Laughon K., Bloom T. (2007). Intimate Partner Homicide, Review and Implications of Research and Policy, Trauma, Violence, \& Abuse, 8, 246-ss.

Campbell J. C., Webster D., Koziol-McLain J., Block C. et alt., (2003), Risk Factors for Femicide in Abusive Relationships: Results From a Multisite Case Control Study, American Journal of Public Health; Vol. 93, Issue 7, 1089-1097.

Campbell, J.C., Webster, D., Koziol-McLain, J., et.al. (2003), Assessing risk factors for intimate partner homicide. National Institute of Justice Journal (250), 14-19. (http://ncjrs.org/pdffiles1/jr000250e.pdf)

Crawley M. J. (2009) The R Book. Wiley; W. N. Venables and B.D. Ripley (2002) Modern Applied Statistics with S. Springer.

Dubugras B., Guevara W. (2007). Homicidio seguido de suicidio, Universitas Psychologica, vol. 6, núm. 2, 231-244.

Kachur SP, Potter LB, James SP, Powell KE. (1995) Suicide in the United States, 19801992. Atlanta: Centers for Disease Control and Prevention, National Center for Injury Prevention and Control, Violence Surveillance Summary, No.1.

Lebart, L., Morineau, A. and Piron, M. (1995) Statistique exploratoire multidimensionnelle, Dunod.

Logan J., Hill H. A. Black M. L., Crosby A. E., Karch D. L, Barnes J. D., Lubell K. M. (2008). Characteristics of Perpetrators in Homicide-Followed-by-Suicide Incidents: National Violent Death Reporting System 17 US States, 2003-2005, American Journal of Epidemiology, 168(9), 1056-1064.

Lund L. E., Smorodinsky S. (2001). Violent Death Among Intimate Partners: A Comparison of Homicide and Homicide Followed by Suicide in California, Suicide and Life-Threatening Behavior, Vol. 31, 4, 451-459.

Menninger, K. Eros e Tanatos (1970), o homem contra si próprio. São Paulo: Ibrasa.

Milroy MD, FRCPath, DMJ. (1998), Homicide followed by suicide: remorse or revenge?, Journal of Clinical Forensic Medicine, Volume 5, Issue 2, 61-64.

Saridakis G. (2004). Violent crime in the United States of America: a time-series analysis between 1960-2000, European Journal of Law and Economics, 203221.

Van Wormer K. (1995), The Dynamics of Murder-Suicide in Domestic Situations, Brief Treatment and Crisis Intervention, Volume 8, Number 3, 274-282.

Vives-Cases, C. Torrubiano-Domínguez J., Álvarez-Dardet C. (2009). The effect of television news items on intimate partner violence murders, The European Journal of Public Health Advance Access, June 23, 1-5.

Voigt L. (2007), Homicide Followed by Suicide. An Integrated Theoretical Perspective Dee Wood Harper, Homicide Studies Volume 11, 295-318. 
Javier G. Fernández Teruelo

Profesor Titular de Derecho penal

Director del Título propio de Criminología de la UO

Sub-Director del Departamento de Ciencias Jurídicas Básicas

Facultad de Derecho. Universidad de Oviedo

$\square$ Campus Universitario de "El Cristo" s/n. 33006-Oviedo

$.985-104721$

$\square 985-104710$

$\square$ xteruelo@uniovi.es

Datos biográficos: Es profesor Titular de Derecho penal y criminología en la Universdad de Oviedo; Director del Título de Criminología de la Universidad de Oviedo, Magistrado Suplente en la Audiencia Provincial de Asturias; cuenta con dos sexenios de investigación; Investigador Principal (IP) de Proyectos de Investigación Nacionales del MEC (DER 2008-5588), autor de 4 monografías y más de 40 artículos doctrinales, casi 100 conferencias, múltiples estancias de investigación en Universidades alemanas e italianas; responsable de contenidos y profesor del Master de Derecho constitucional de la UCA de El Salvador, formador de jueces de la Agencia Española de Cooperación Internacional e Bolivia, etc. 